\title{
Absence of radiative corrections in four-dimensional topological Yang-Mills theories
}

\author{
O. C. Junqueira, ${ }^{1, *}$ A. D. Pereira, ${ }^{2, \dagger}$ G. Sadovski, ${ }^{1, \$}$ R. F. Sobreiro, ${ }^{1, \S}$ and A. A. Tomaz ${ }^{3,4, \|}$ \\ ${ }^{1}$ UFF - Universidade Federal Fluminense, Instituto de Física, \\ Avenida Litorânea s/n, 24210-346 Niterói, Rio de Janeiro, Brazil \\ ${ }^{2}$ Institute for Theoretical Physics, University of Heidelberg, \\ Philosophenweg 12, 69120 Heidelberg, Germany \\ ${ }^{3}$ CBPF - Centro Brasileiro de Pesquisas Físicas, Rua Dr. Xavier Sigaud 150, \\ 22290-180, Rio de Janeiro, RJ, Brazil \\ ${ }^{4} U F A B C$ - Universidade Federal do ABC, Centro de Matemática, Computação e Cognição, \\ 09210-580 Santo André, São Paulo, Brazil
}

(Received 1 June 2018; published 16 July 2018)

\begin{abstract}
We prove that all connected $n$-point Green functions of four-dimensional topological Yang-Mills theories quantized in the (anti-)self-dual Landau gauges are tree-level exact, i.e., there are no radiative corrections in this gauge choice.
\end{abstract}

DOI: 10.1103/PhysRevD.98.021701

\section{INTRODUCTION}

Topological Yang-Mills theories are, essentially, background independent gauge theories which only depend on global degrees of freedom [1-4]. The first ideas about topological solutions from self-dual Yang-Mills equations go back to [5], where topological solutions known as instantons were used to explain the $U(1)$ problem in nonAbelian theories [6]. Another interesting four-dimensional theory is the so-called Donaldson-Witten topological quantum field theory, which can be taken as a tool to compute the Donaldson topological invariants $[1,7,8]$. Related to the latter, it was discussed in [2] that Donaldson-Witten's theory can be recovered from a particular gauge fixing of an action which is a pure topological invariant. The gauge fixing of four-dimensional topological gauge theories was also investigated in [9]. The particular choice of (anti-)self-dual Landau gauges [(A)SDLG] was first studied in [10]. The renormalizability of the theory quantized in this gauge was investigated in $[10,11]$, where the algebraic renormalization technique $[12,13]$ was employed. It was verified that, due to the rich set of Ward identities in this gauge, there is only one independent renormalization parameter. Moreover, all

\footnotetext{
*octavio@if.uff.br

a.pereira@thphys.uni-heidelberg.de

¥gsadovski@id.uff.br

\$rodrigo_sobreiro@id.uff.br

tomaz@cbpf.br
}

Published by the American Physical Society under the terms of the Creative Commons Attribution 4.0 International license. Further distribution of this work must maintain attribution to the author(s) and the published article's title, journal citation, and DOI. Funded by SCOAP. propagators were shown to be tree-level exact (as well as all 1PI two-point Green functions). In particular, it was proven that the gauge propagator and the vacuum polarization vanish to all orders of perturbation theory, see [11].

The aim of the present paper is to improve the understanding of quantum four-dimensional topological YangMills theory in the (A)SDLG. Specifically, we show that all connected $n$-point Green functions are tree-level exact, i.e., that all connected $n$-point Green functions of the theory do not receive any radiative corrections. For what follows, the fact that the gauge field propagator vanishes to all orders in perturbation theory and concepts of Becchi-Rouet-StoraTyutin (BRST) cohomology are pivotal.

This work is organized as follows: In Sec. II we provide an overview of four-dimensional topological Yang-Mills theories quantized in the (A)SDLG. Section III is devoted to the proof of the tree-level exactness of all connected $n$-point Green functions and, finally, Sec. IV contains our final considerations.

\section{TOPOLOGICAL GAUGE THEORIES AT THE (ANTI-)SELF-DUAL LANDAU GAUGES}

Topological Yang-Mills theories ${ }^{1}$ in four-dimensional Euclidean spacetime ${ }^{2}$ can be defined by a topological

\footnotetext{
${ }^{1}$ It is worth mentioning that the action $S_{0}(A)$ encompasses a wide range of topological gauge theories. The Pontryagin action is the most common case because it can be defined for all semisimple Lie groups. Nevertheless, other cases can also be considered. For instance, Gauss-Bonnet and Nieh-Yang topological gravities can be formulated for orthogonal groups [14].

${ }^{2}$ In this work, we consider flat Euclidean spacetime. Although the topological action is background independent, the gaugefixing term entails the introduction of a background. Ultimately, background independence is recovered at the level of correlation function due to BRST symmetry [2-4].
} 
invariant action, $S_{0}[A]$, where $A_{\mu}^{a}$ is the gauge field algebravalued in a semisimple Lie group $G$. As discussed in [10], a topological Yang-Mills theory carries three independent gauge symmetries, namely,

$$
\begin{aligned}
\delta A_{\mu}^{a} & =D_{\mu}^{a b} \alpha^{b}+\alpha_{\mu}^{a}, \\
\delta F_{\mu \nu}^{a} & =-g f^{a b c} \alpha^{b} F_{\mu \nu}^{c}+D_{\mu}^{a b} \alpha_{\nu}^{b}-D_{\nu}^{a b} \alpha_{\mu}^{b}, \\
\delta \alpha_{\mu}^{a} & =D_{\mu}^{a b} \lambda^{b},
\end{aligned}
$$

where $D_{\mu}^{a b}=\delta^{a b} \partial_{\mu}-f^{a b c} A_{\mu}^{b}$ is the covariant derivative in the adjoint representation of $G, F_{\mu \nu}^{a}=\partial_{\mu} A_{\nu}^{a}-\partial_{\nu} A_{\mu}^{a}+$ $g f^{a b c} A_{\mu}^{b} A_{\nu}^{c}$ is the field strength and $g$ is the coupling parameter. The parameters $\alpha_{\mu}^{a}, \alpha^{a}$ and $\lambda^{a}$ are $G$-valued gauge parameters. Thus, in order to quantize the theory, three gauge constraints are needed. The BRST quantization will be employed for this purpose.

The BRST procedure $[2,12,13]$ starts by promoting the gauge parameters to ghost fields: $\alpha_{\mu}^{a} \rightarrow \psi_{\mu}^{a}, \alpha^{a} \rightarrow c^{a}$, and $\lambda^{a} \rightarrow \phi^{a}$, and in the definition of a nilpotent BRST symmetry,

$$
\begin{aligned}
s A_{\mu}^{a} & =-D_{\mu}^{a b} c^{b}+\psi_{\mu}^{a}, \\
s c^{a} & =\frac{g}{2} f^{a b c} c^{b} c^{c}+\phi^{a}, \\
s \psi_{\mu}^{a} & =g f^{a b c} c^{b} \psi_{\mu}^{c}+D_{\mu}^{a b} \phi^{b}, \\
s \phi^{a} & =g f^{a b c} c^{b} \phi^{c} .
\end{aligned}
$$

In (2.4), $c^{a}, \psi_{\mu}^{a}$ and $\phi^{a}$ are, respectively, the Faddeev-Popov ghost field, the topological ghost field and the bosonic ghost field, while $s$ is the nilpotent BRST operator.

The gauge choice we employ in this work is the (anti-) self-dual Landau gauges [10,11], defined by

$$
\begin{aligned}
\partial_{\mu} A_{\mu}^{a} & =0, \\
F_{\mu \nu}^{a} \pm \tilde{F}_{\mu \nu}^{a} & =0, \\
\partial_{\mu} \psi_{\mu}^{a} & =0,
\end{aligned}
$$

where $\tilde{F}_{\mu \nu}^{a} \equiv \frac{1}{2} \epsilon_{\mu \nu \alpha \beta} F_{\alpha \beta}^{a}$ is the dual field strength. To enforce such constraints, three BRST doublets are needed:

$$
\begin{aligned}
s \bar{c}^{a} & =b^{a}, & s b^{a} & =0, \\
s \bar{\chi}_{\mu \nu}^{a} & =B_{\mu \nu}^{a}, & s B_{\mu \nu}^{a} & =0, \\
s \bar{\phi}^{a} & =\bar{\eta}^{a}, & s \bar{\eta}^{a} & =0,
\end{aligned}
$$

where $\bar{\chi}_{\mu \nu}^{a}$ and $B_{\mu \nu}^{a}$ are (anti-)self-dual fields, according to the positive (negative) sign in the second condition in (2.5). In (2.6), the fields $b^{a}, B_{\mu \nu}^{a}$ and $\bar{\eta}^{a}$ are the Lautrup-Nakanishi fields which implement the gauge conditions (2.5) while $\bar{c}^{a}, \bar{\chi}_{\mu \nu}^{a}$ and $\bar{\phi}^{a}$ are the Faddeev-Popov, topological and bosonic anti-ghost fields, respectively. For completeness, the quantum numbers of all fields are displayed in Table I.

The complete gauge fixing action in the gauge (2.5) takes the form

$$
\begin{aligned}
S_{g f}= & s \int d^{4} z\left[\bar{c}^{a} \partial_{\mu} A_{\mu}^{a}+\frac{1}{2} \bar{\chi}_{\mu \nu}^{a}\left(F_{\mu \nu}^{a} \pm \tilde{F}_{\mu \nu}^{a}\right)+\bar{\phi}^{a} \partial_{\mu} \psi_{\mu}^{a}\right] \\
= & \int d^{4} z\left[b^{a} \partial_{\mu} A_{\mu}^{a}+\frac{1}{2} B_{\mu \nu}^{a}\left(F_{\mu \nu}^{a} \pm \tilde{F}_{\mu \nu}^{a}\right)+\left(\bar{\eta}^{a}-\bar{c}^{a}\right) \partial_{\mu} \psi_{\mu}^{a}+\bar{c}^{a} \partial_{\mu} D_{\mu}^{a b} c^{b}\right. \\
& \left. \pm \frac{1}{2} g f^{a b c} \bar{\chi}_{\mu \nu}^{a} c^{b}\left(F_{\mu \nu}^{c} \pm \tilde{F}_{\mu \nu}^{c}\right)-\bar{\chi}_{\mu \nu}^{a}\left(\delta_{\mu \alpha} \delta_{\nu \beta} \pm \frac{1}{2} \epsilon_{\mu \nu \alpha \beta}\right) D_{\alpha}^{a b} \psi_{\beta}^{b}+\bar{\phi}^{a} \partial_{\mu} D_{\mu}^{a b} \phi^{b}+g f^{a b c} \bar{\phi}^{a} \partial_{\mu}\left(c^{b} \psi_{\mu}^{c}\right)\right] .
\end{aligned}
$$

The full action $S\left[\Phi_{i}\right]$, for all fields $\Phi_{i} \equiv\{A, \psi, \bar{\chi}, c, \bar{c}$, $\phi, \bar{\phi}, \eta, \bar{\eta}, B, b\}$, is then composed by a sum of a topological invariant term and a BRST-exact one,

$$
S=S_{0}+S_{g f}
$$

The action (2.8) has a few interesting quantum properties [11]:

TABLE I. Canonical dimension and ghost number of the fields.

\begin{tabular}{lcccccccccc}
\hline \hline Field & $A$ & $\psi$ & $c$ & $\phi$ & $\bar{c}$ & $b$ & $\bar{\phi}$ & $\bar{\eta}$ & $\bar{\chi}$ & $B$ \\
\hline Dim & 1 & 1 & 0 & 0 & 2 & 2 & 2 & 2 & 2 & 2 \\
Ghost $n^{o}$ & 0 & 1 & 1 & 2 & -1 & 0 & -2 & -1 & -1 & 0 \\
\hline \hline
\end{tabular}

(i) It is renormalizable to all orders in perturbation theory. Moreover, due to the rich set of Ward identities displayed by the action (2.8), the most general counterterm carries only one independent renormalization parameter, denoted by $a$ :

$$
\Sigma^{c}=a \int d^{4} x\left(B_{\mu \nu}^{a} F_{\mu \nu}^{a}-2 \bar{\chi}_{\mu \nu}^{a} D_{\mu}^{a b} \psi_{\nu}^{b}-g f^{a b c} \bar{\chi}_{\mu \nu}^{a} c^{b} F_{\mu \nu}^{c}\right) .
$$

(ii) All propagators are tree-level exact, which means that, in the (A)SDLG, they do not receive radiative corrections.

(iii) In particular, the gauge field propagator vanishes exactly in the (A)SDLG: 


$$
\left\langle A_{\mu}^{a} A_{\nu}^{b}\right\rangle(p)=0
$$

In the following, we collect the Feynman rules derived from (2.8). The relevant propagators are represented by ${ }^{3}$ :

$$
\begin{aligned}
& \langle A A\rangle=\text { eеeеe }, \quad\langle c \bar{c}\rangle=\ldots \ldots \ldots . . \quad, \quad\langle\bar{\chi} \psi\rangle=\text { mm }, \quad\langle A b\rangle=\text { eee- }, \\
& \langle\bar{\eta} \psi\rangle=\ldots m, \quad\langle A B\rangle=\text { eе }=, \quad\langle\phi \bar{\phi}\rangle=: \ldots: \cdots: !: .
\end{aligned}
$$

The relevant vertexes are represented by:
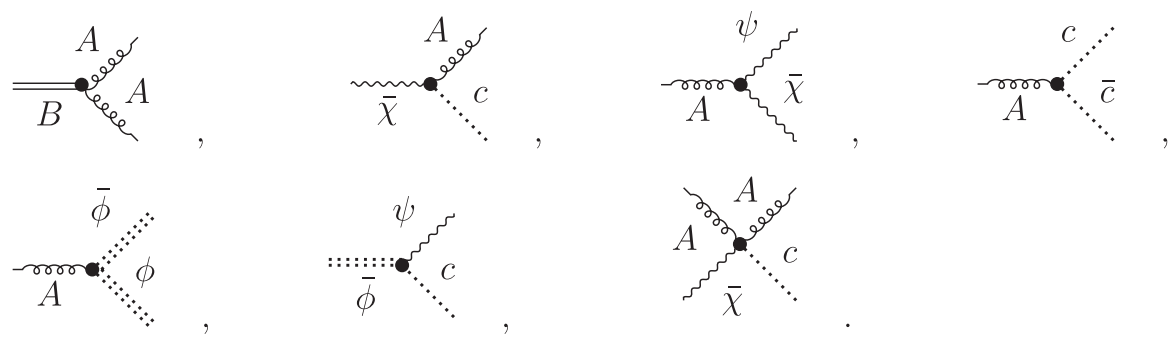

In principle we do not have to include the gauge propagator in (2.11) - which is null-but this will be necessary to visualize the tree-level exactness of the theory, since such a propagator, as discussed later on, is required to close loops, leading to vanishing diagrams at the quantum level.

\section{ABSENCE OF RADIATIVE CORRECTIONS}

To show that the action (2.8) defines a theory free of radiative corrections, it is convenient to split the argumentation into propositions.

Proposition 1: Any connected loop diagram containing an internal $A$-leg vanishes unless the branch generated by the $A$-leg ends up in external $B$ - or $b$-legs.

Proof.-To prove this proposition, we must consider a combination of two facts: (1) $\langle A A\rangle=0$ to all orders and (2) the gauge field only propagates through the nonvanishing mixed propagators $\langle B A\rangle$ and $\langle b A\rangle$. Hence, from an internal $A$-leg arising from an arbitrary vertex, denoted by a black dot, aee, we only have two possibilities: aeenand aee = In the same way, the fields $B$ and $b$ only propagate through $A$. Graphically, we now havereen $\bullet$ and oee $=$. Nonetheless, the former is not at our disposal since there is no vertex containing $b$, vide (2.12). The latter, on the other hand, must be a $B A A$ vertex since it is the only one containing $B$. Thus, an internal $A$-leg in any loop diagram will propagate to $B$ and the latter will end up in a $B A A$ vertex,

\footnotetext{
${ }^{3}$ From (2.7), a $\bar{c} \psi$ mixed propagator also seems to be relevant. However, this term can easily be eliminated by a trivial-Jacobian redefinition of the $\bar{\eta}$ field given by $\bar{\eta} \rightarrow \bar{\eta}+\bar{c}$.
}

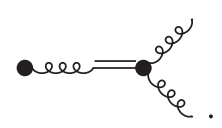

Applying the above reasoning for the two newly created $A$-legs, we end up with two more $B A A$ vertexes and four $A$-legs. Since the number of $A$-legs only increases, we can continue this process ad infinitum leading to a cascade effect of exponential proliferation of $A$-legs:

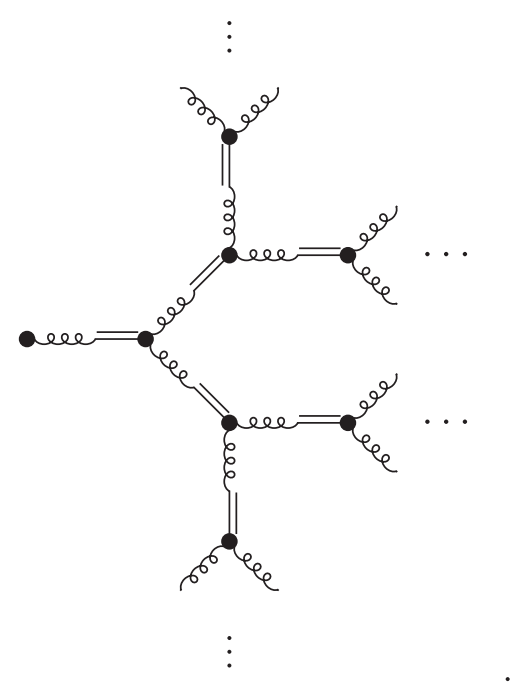

There are three possibilities here: (1) trying to close a loop in the diagram (3.2) requires an $\langle A A\rangle$ internal propagator, which would result in a vanishing diagram; (2) to consider external $A$-legs, which also requires a $\langle A A\rangle$ propagator, resulting in a vanishing diagram and; (3) one could consider that all remaining $A$-legs end up in external $B$ - or $b$-legs.

We should note that all vertexes, except one, present in (2.8) contain at least one $A$-leg, therefore the cascade effect 
always occur for these cases. The only exception is the vertex $\bar{\phi} c \psi$.

Corollary 1.1: In a connected loop diagram, any branch arising from the vertex $\bar{\phi} c \psi$ results in a vanishing diagram unless this branch ends up in external $B$ - or $b$-legs.

Proof.-Let us start with the vertex of interest, i.e., $\bar{\phi} c \psi$. To construct a loop diagram from this three-vertex we have to propagate it to another vertex. The $\bar{\phi}$-leg could only propagate to the vertex $\bar{\phi} A \phi$ through $\langle\bar{\phi} \phi\rangle$; the $c$-leg only to $\bar{c} A c$ through $\langle\bar{c} c\rangle$ and; the $\psi$-leg to the vertexes $\bar{\chi} A \psi, \bar{\chi} c A$ or $\bar{\chi} c A A$ through $\langle\psi \bar{\chi}\rangle(\langle\bar{\eta} \psi\rangle$ is not considered because there is no vertex containing $\bar{\eta}$ ). Graphically, the possibilities of completing the legs arising from this vertex are

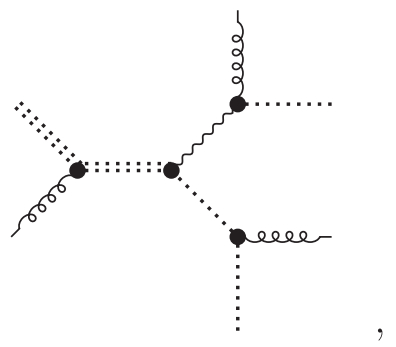

But all possible branches contain at least one remaining $A$-leg. By evoking Proposition 1, the proof is completed.

Corollary 1.2: Any connected loop diagram containing a $\left(\Phi_{i} \neq\{B, b\}\right)$-external leg vanishes.

Proof.-There are two steps toward this proof: (1) consider the external leg joined to a vertex containing an $A$ field. In this case, $A$ is an internal leg. Thus, Proposition 1 takes place and the graph either vanishes or generates a branch with external $B$ - or $b$-legs and no loop can be constructed; (2) now, consider the external leg joined to a vertex not containing $A$, i.e., the vertex $\bar{\phi} c \psi$. The field $\bar{\phi}$ only propagates through $\langle\bar{\phi} \phi\rangle, c$ through $\langle\bar{c} c\rangle$, and $\psi$ only through $\langle\bar{\chi} \psi\rangle$ or $\langle\bar{\eta} \psi\rangle$. For this reason, it is impossible to propagate the vertex $\bar{\phi} c \psi$ to another vertex $\bar{\phi} c \psi$. In other words, from the vertex $\bar{\phi} c \psi$, we should necessarily propagate it to the vertexes containing an $A$ field. Now, Corollary 1.1 takes place and the graph, again, either vanishes or generates a branch with external $B$ - or $b$-legs and no loop can be constructed.

Proposition 2: Any connected $n$-point function of the form $\left\langle B\left(x_{1}\right) B\left(x_{2}\right) \ldots b\left(x_{n-1}\right) b\left(x_{n}\right)\right\rangle$ vanishes.

Proof.-Due to (2.6), and the fact that expectation values of any BRST-exact terms vanish. One can write these $n$-functions as BRST-exact correlators, namely

$$
\langle B B B \ldots b b\rangle=\langle s \bar{\chi} B B \ldots b b\rangle=\langle s(\bar{\chi} B B \ldots b b)\rangle=0,
$$

and

$$
\langle B B B \ldots b b\rangle=\langle B B \ldots s \bar{c} b\rangle=\langle s(B B B \ldots \bar{c} b b)\rangle=0,
$$

which vanish due to BRST-invariance.

Proposition 3: All connected n-point Green functions are tree-level exact.

Proof.-Let us take a connected loop diagram with $n$ external legs with arbitrary fields $\Phi_{i}$. From Corollary 1.2, if there is at least one field different from $B$ or $b$, the graph either vanishes or is a tree-level graph. Then, there remains the possibility of a graph with $n$ external legs formed by $B$ or $b$ fields. In this case Proposition 2 takes over and the Green function $\langle B B \ldots b b\rangle$ vanishes, meaning that this Green function is zero and receive no radiative corrections. Hence, all connected $n$-point Green functions are tree-level exact.

\section{CONCLUSIONS}

In this work we have considered four-dimensional topological Yang-Mills theories quantized in the (anti-)self-dual Landau gauges $[1,2,7,8,10,11]$, which is renormalizable to all orders in perturbation theory $[10,11]$. This particular gauge choice displays a rich set of symmetries, which implies on a counterterm containing only one independent renormalization parameter, see [11]. Moreover, the gauge propagator vanishes to all orders.

The fact that the gauge propagator vanishes exactly was employed to show the main result of this paper: All connected n-point Green functions of four-dimensional topological gauge theories quantized in the (anti-)self-dual Landau gauges are tree-level exact. This means that, in this gauge, the theory remains "classical" because there are no radiative corrections to be considered. This is a very interesting, yet subtle, result. The subtlety lives on the fact that the theory is not finite (there is a nontrivial counterterm to be included in order to absorb the divergences of the theory [11]) but the divergences are canceled out due to the vanishing of the gauge propagator which is always needed in order to close a loop diagram or due to the BRST symmetry.

It is worth mentioning that the topological gauge theory considered here is essentially of the Donaldson-Witten type [1] formulated in a different gauge choice [2]. Since Donaldson-Witten theory is related to $N=2$ Wess-Zumino supersymmetric theory via a twist [15], it could be interesting to understand the corresponding 
supersymmetric theory in different gauge choices such as the (A)SDLG. Specifically, the consequences of the absence of radiative corrections in the (A)SDLG could imply in some interesting features of its supersymmetric counterpart. Moreover, supersymmetric formulations of the BRST transformations (2.4) and (2.6), as in for instance $[16,17]$, could also enlighten such investigation.

\section{ACKNOWLEDGMENTS}

The Conselho Nacional de Desenvolvimento Científico e Tecnológico (CNPq-Brazil) and the Coordenação de Aperfeiçoamento de Pessoal de Nível Superior (CAPES) are acknowledged for financial support. A. D. P. acknowledges funding by the DFG, Grant No. Ei/1037-1.
[1] E. Witten, Topological quantum field theory, Commun. Math. Phys. 117, 353 (1988).

[2] L. Baulieu and I. Singer, Topological Yang-Mills symmetry, Nucl. Phys. B, Proc. Suppl. 5, 12 (1988).

[3] M. Blau and G. Thompson, Do metric independent classical actions lead to topological field theories?, Phys. Lett. B 255, 535 (1991).

[4] M. Abud and G. Fiore, Batalin-Vilkovisky approach to the metric independence of TQFT, Phys. Lett. B 293, 89 (1992).

[5] A. A. Belavin, A. M. Polyakov, A. S. Schwartz, and Y. S. Tyupkin, Pseudoparticle solutions of the Yang-Mills equations, Phys. Lett. 59B, 85 (1975).

[6] G. 't Hooft, Computation of the quantum effects due to a fourdimensional pseudoparticle, Phys. Rev. D 14, 3432 (1976).

[7] R. Brooks, D. Montano, and J. Sonnenschein, Gauge fixing and renormalization in topological quantum field theory, Phys. Lett. B 214, 91 (1988).

[8] D. Birmingham, Topological field theory, Phys. Rep. 209, 129 (1991).

[9] R. Myers, Gauge fixing topological Yang-Mills, Int. J. Mod. Phys. A 05, 1369 (1990).

[10] A. Brandhuber, O. Moritsch, M. de Oliveira, O. Piguet, and M. Schweda, A renormalized supersymmetry in the topological Yang-Mills field theory, Nucl. Phys. B431, 173 (1994).
[11] O. C. Junqueira, A. D. Pereira, G. Sadovski, R. F. Sobreiro, and A. A. Tomaz, Topological Yang-Mills theories in selfdual and anti-self-dual Landau gauges revisited, Phys. Rev. D 96, 085008 (2017).

[12] L. Baulieu and J. Thierry-Mieg, The principle of BRS symmetry: An alternative approach to Yang-Mills theories, Nucl. Phys. B197, 477 (1982).

[13] O. Piguet and S. P. Sorella, Algebraic Renormalization, Lecture Notes in Physics Monographs (Springer, Berlin, Heidelberg, 1995), Vol. 28.

[14] A. Mardones and J. Zanelli, Lovelock-Cartan theory of gravity, Classical Quantum Gravity 8, 1545 (1991).

[15] F. Fucito, A. Tanzini, L. C. Q. Vilar, O. S. Ventura, C. A. G. Sasaki, and S. P. Sorella, Algebraic renormalization: Perturbative twisted considerations on topological YangMills theory and on $\mathrm{N}=2$ supersymmetric gauge theories, arXiv:hep-th/9707209.

[16] J. L. Boldo, C. P. Constantinidis, F. Gieres, M. Lefrancois, and O. Piguet, Observables in topological Yang-Mills theories, Int. J. Mod. Phys. A 19, 2971 (2003).

[17] J. L. Boldo, C. P. Constantinidis, O. Piguet, F. Gieres, and M. Lefrançois, Topological Yang-Mills theories and their observables: A superspace approach, Int. J. Mod. Phys. A 18, 2119 (2003). 